\title{
An Extremely Giant Lumbar Schwannoma: New Classification (Kotil) and Mini-Open Microsurgical Resection
}

\author{
Kadir Kotil \\ Department of Neurosurgery, Istanbul Arel University, Tepekent, Istanbul, Turkey
}

Purely extradural giant lumbar schwannomas are rare lesions. Classification of these tumors is performed according to the sizes and spills and is named the modified Sridhar's classification. The management of these tumors has traditionally been performed by combined antero-posterior large or two-stage exposures. This combined exposure has many disadvantages. Recent reports have demonstrated the safety and efficacy of removal of these tumors using mini-open micro surgical resection for the schwannomas, but the safety and efficacy have not been established for extremely large giant tumors. We report a case of a giant L 4 dumb-bell schwannoma successfully resected through a mini-open micro surgical resection. These neoplasms can be safely and effectively treated with mini-open techniques. Reduction in blood loss, hospitalization, and tissue disruption may be potential benefits of this approach. We discuss the technical details of this surgical approach along with the limitations and possible complications. In addition, this study is included in the current schwannoma (Kotil) classification.

Keywords: Extradural; Schwannoma; Microsurgical resection; Miniopen; Lumbar; New classificaiton

\section{Introduction}

Schwannomas are the most common form of intradural spinal tumor, making up almost one-third of primary spinal neoplasms [1,2]. Extradural forms are rare neoplasms, and in addition, giant dumb-bell schwannomas, which extend to anterior vascular structures, are extremely rare [3,4]. Love and Dodge [5] in 1952 first coined the term dumb-bell tumor, reporting a neurogenic tumor with in growths into the spinal canal. In addition, these tumors may grow extensively outside the spinal canal and compress the paraspinal-iliopsoas complexes as well as retroperitoneal vascular structures. Various approaches have been described for treating these tumors $[1,2,4,6$ -
11]. The combined antero-posterior approach has mostly been used but it carries the disadvantage of being a twostage operation [7,9]. Radical resections are needed with both the retroperitoneal and posterior spinal approaches [12]. The mainstay of the posterior approach is gross total resection, classically involving midline or paramedian incisions, bilateral subperiosteal muscle stripping, extensive facetectomy and radical foraminotomy. Fusion is often warranted in order to prevent deformity, pain, and neurological deterioration [3,7,9]. In addition, in our case, the present tumor could not be classified using the modified Sridhar's classification [10] due to both the tumor size and the extension of the tumor to neurovascular structures. We describe an alternative approach, called in-

Received Feb 28, 2013; Revised Jun 3, 2013; Accepted Sep 1, 2013

Corresponding author: Kadir Kotil

Department of Neurosurgery, Istanbul Arel University,

Turkoba mahhalesi erguvan sokak no:26 34537-tepekent-Buyukçekmece, Istanbul, Turkey

Tel: +90-216-360-12-26, Fax: +90-216-302-03-91, E-mail: kadirkotil@gmail.com 
termuscular mini-open micro surgical resection, for onestage removal of extremely giant dumb-bell-shaped spinal tumors. This approach, firstly, developed by Kadir Kotil and not previously reported, provides the simplest and most direct route for resection of giant dumb-bell tumors.

\section{Case Report}

A 37-year-old woman presented with a history of chronic right leg pain, paresthesia and minimal distal right leg weakness. Magnetic resonance imaging (MRI) scanning revealed a large dumbbell-shaped extradural foraminal lesion at the L4-L5 level with significant major vascular extension to structures such as the aorta and inferior vena cava (Fig. 1). A preoperative biopsy was made by a spinal needle percutaneously, and the pathology confirmed the lesion to be a benign schwannoma. The patient was prepared for both the anterior and posterior approaches,
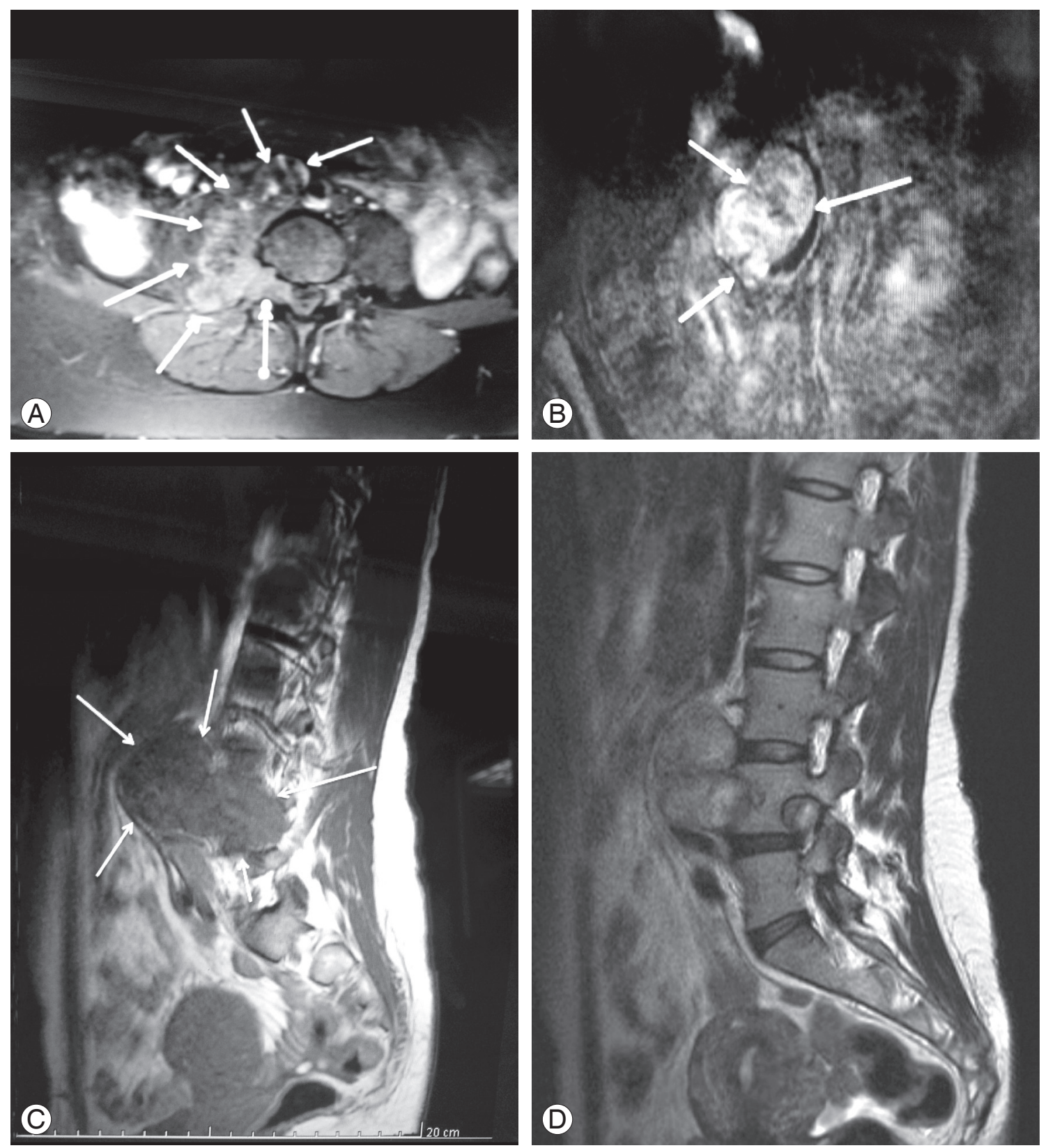

Fig. 1. (A) T2-weighted axial image showing a giant dumb-bell-shaped mass extending anteriorly. (B) Post-gadolinium T1W coronal image displaying a heterogenously enhancing giant dumb-bell mass, and lateral displacement of the neurovascular structures. (C) Lateral T2-weighted image showing the horizontal extent of the lesion and (D) widening of the neural foramina. 
and the vascular surgeon waited for us in the operation room to carry out plan B (for the vascular complications). On the right side, a $3.5 \mathrm{~cm}$ paramedian incision was made. Two Meyerding retractors were placed into the intermuscular septum. The spinous processes, facet and interspinous ligaments were preserved, exposing the intra-foraminal component of the dumb-bell tumor. We exposed the intra-canalicular component, which was excised using microsurgical techniques. It was possible to preserve the functioning nerve root as only rootlets were embedded in the capsule of the tumor. No other nerve root was sacrificed. Once a generous internal decompression was accomplished, the capsule of the tumor was mobilized. There was a well-defined surgical plane between the tumor capsule and the adjoining vascular structure. Blunt dissection was performed in the surgical plane as the tumor was well separated from major neurovascular structures by the psoas muscle. The tumor was removed piece by piece. There was no invasion of anterior vascular structures and the tumor was easily removed. Postoperatively, the patient's symptoms resolved and she was discharged from the hospital on the first postoperative day. The postoperative MRI showed no residual tumor (Fig. 2). There were no skin problems (Fig. 3). The patient returned to normal activities after 1 week and was asymptomatic with no neurological deficits at the final 3 month follow-up.

\section{Classification}

Sridhar [10] was the first, in 2001, to suggest a classifica- tion system of benign spinal schwannoma including giant and invasive spinal schwannomas (type I to V) (Table 1). Afterwards, Park et al. [8] recently reported the use of a new classification system, and Type VI and Type VII were added. But the classification system as defined by Park et al. [8] were inadequate because both the figures and the tumors were not clearly described in their manuscript. However, the present tumor also had a large extradural component which extended to neurovascular structures and it passed to the anterior vertebral column due to the extremely large size of this giant schwannoma. Thus, this tumor could not be properly classified based on Sridhar's classification. If the tumor extends to anterior neurovascular structures in the retroperitoneal plane, the tumor could be type VIII (Table 1, Fig. 4). Our case could not be classified based on Sridhar's spinal schwannoma classification system. Thus, as shown in our case, type VIII must be added to the modified Sridhar classification (Kotil

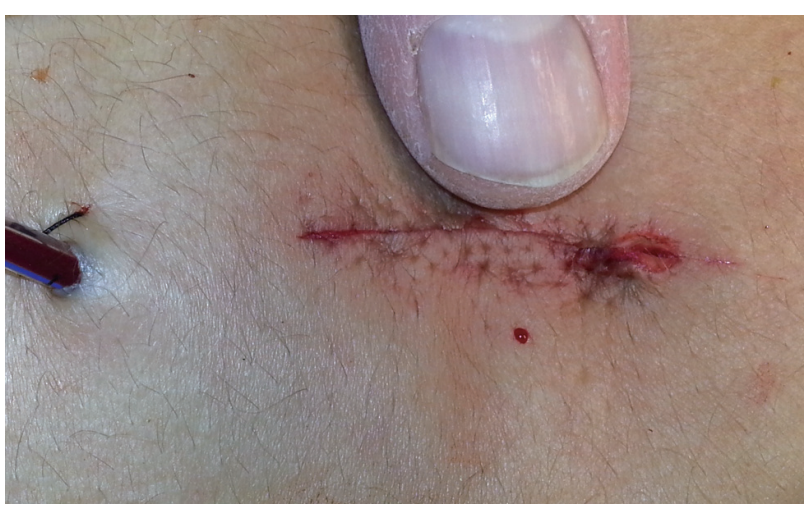

Fig. 3. After complete excision and skin closure.

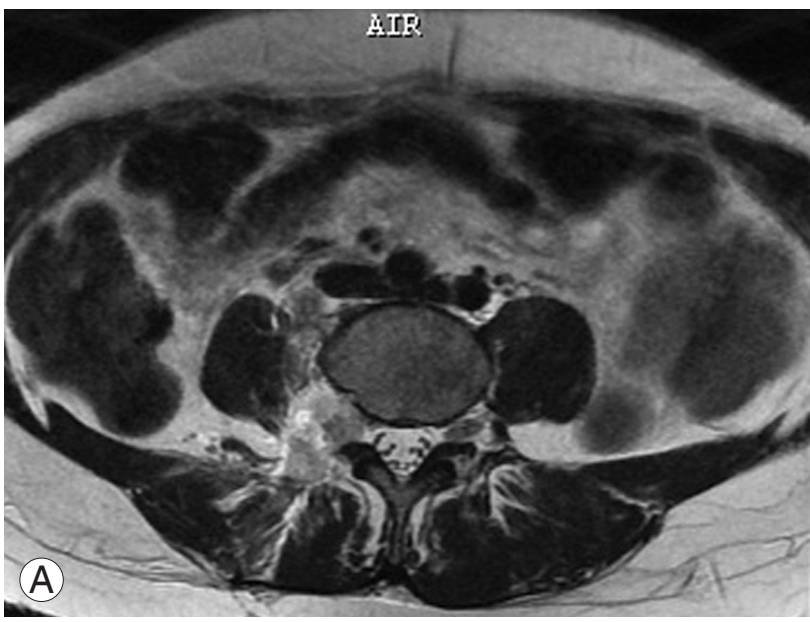

Fig. 2. Postoperative, contrast-enhanced axial (A) and sagittal (B) magnetic resonance imaging images showing complete excision.

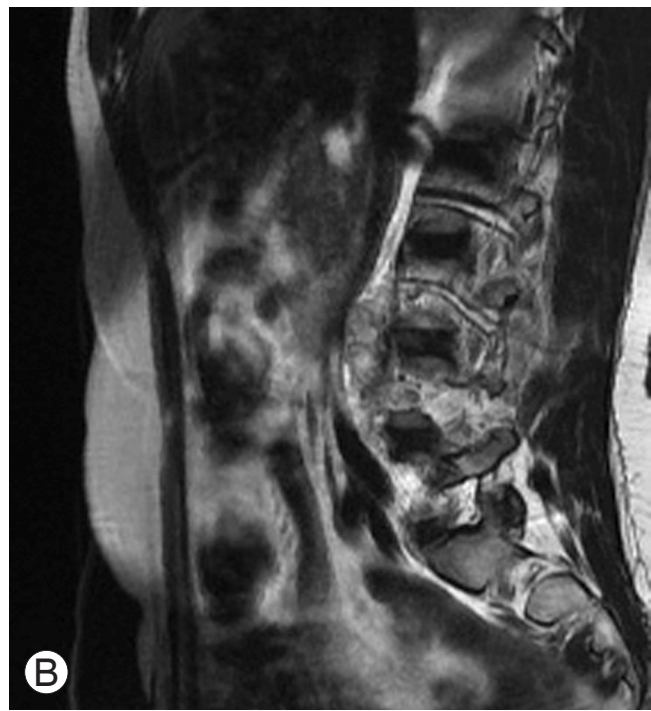


Table 1. A new classification of bening nerve sheat tumors of Kotil (Modified Sridhar Classification of bening nerve sheat tumors [Kotil classification])

\begin{tabular}{ll}
\hline Type I & Intraspinal tumor, <2 vertebral segments in length \\
& a: intradural; \\
& b: extradural \\
Type II & Intraspinal tumor $>2$ vertebral segments in length (giant tumor) \\
\hline Type III & Intraspinal tumor/extention into nerve root foramen \\
Type IV & Intraspinal tumor/extraspinal extention (dumbbell tumors): \\
& a: extraspinal component $<2.5 \mathrm{~cm}$; \\
& b: extraspinal component $>2.5 \mathrm{~cm}$ (giant tumor) \\
Type V & Tumor w/erosion into the vertebral bodys (giant invasive tumor), lateral \& posterior extentions into the myofascial planes \\
Type VI & a: Tumor one vertebral levels; \\
& b: extraspinal component $>2.5$ cm (giant tumor); \\
& d: extending into the posterior Myofascial planes; \\
& e: anteriorly, eroding the vertebralbodys to varying extents; and \\
& f: and compression of the anterior neurovascular structure
\end{tabular}

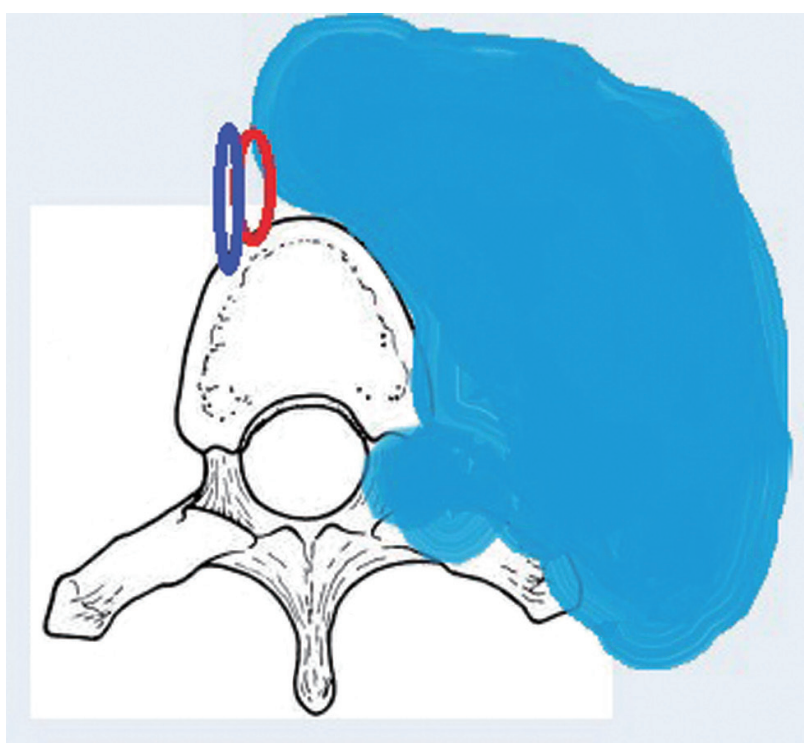

Fig. 4. This medical illustration shows the new classification system. The tumor has displaced the neurovascular structures antero-laterally. Type 5 was defined by Sridhar et al. [10]. Type VI has been added by us.

classification) system of benign spinal schwannomas.

\section{Discussion}

Complex spinal dumb-bell tumors are challenging surgical lesions. Often these tumors will compress paraspinal and iliopsoas muscle complexes as well as vascular structures. The combined antero-posterior approach has normally been used for its treatment. This approach is time consuming and has the disadvantage of the necessity for the two stages of translumbar and retroperitoneal dissec- tion in the operation $[3,4]$. With better understanding of the biomechanics of the spine and the evolution of microsurgical techniques, there has been a resurgence of single stage surgeries, among which the intermuscular exposure provides the simplest and the most direct route for resection of an extremely large giant dumb-bell schwannoma [9].

The surgical complications of schwannomas are reported frequently with intradural tumors [6]. The incidence of neurological deficits is reportedly low after sacrificing the affected nerve root during spinal schwannoma treatment. Although the incidence has been widely reported, the operative method for nerve root resection of these tumors has been not clarified.

Firstly, a retroperitoneal postero-lateral approach is made with a long midline skin incision, unilateral subperiosteal muscle stripping from the posterior spinous elements, laminectomy extending to the levels above and below the tumor and radical facetectomy on the side of the foraminal tumor [4]. Removal of all these structures may result in immediate or delayed instability of the spine, muscular damage, and also exposes the major vascular structure to the possibility of inadvertent injury during surgery [5].

Recently, both minimally invasive and mini-open access were presented in many papers [3,4,6-8], but there was no report on this approach for an extremely large giant dumb-bell tumor. Lu et al. [7] recently reported the use of an alternative approach, encompassing midline mini-open access with expandable tubular retractors. The 18 patients who underwent the mini-open approach had 
reduced blood loss and length of stay as compared to the 9 patients who underwent the standard open technique. However, this approach is not feasible in cases of extradural giant dumb-bell schwannomas that typically extend to vascular structures, such as in our case.

Singh et al. [4] recently reported the use of a new approach, one-stage surgery, that was presented for the treatment of giant dumb-bell schwannomas. Although this approach permits single stage removal of the dumbbell tumor in selected cases, it is a time-consuming and technically demanding technique; additionally, this approach seems to be a very destructive type of surgery, and many complications can be seen with this destructive surgery. The intermuscular mini-open micro surgical resection provides a direct route for the minimal invasive resection of extremely large giant dumb-bell tumors. It provides direct access to the intradural component; hence intradural extension is no constraint for this technique. This approach is based on two concepts. First, there are minimal biomechanical consequences to complete sectioning of the unilateral paraspinal muscles. Second, there is a well-defined surgical plane between the tumor capsule and the adjacent structures. It is important to assess the degree of anterior spinal involvement of the tumor, or to determine whether anterior column stability is present. The intermuscular approach is appropriate in cases where extensive anterior tumor dissection from the spine is required. We advocate the routine use of a CT scan in the preoperative preparation for all dumb-bell tumors. CT is not only superior to MRI in demonstrating bony involvement, but clearly outlines the surgical plane as well [8]. This permits mobilization of the tumor from its surrounding structures and allows the complete removal of the tumor using a single miniopen microsurgical approach. The most difficult part of extra-canalicular capsule dissection occurs at the most anterior part, as this is the last area to be exposed through the intermuscular approach. Total resection of the tumor capsule is recommended, as there is a significant risk of recurrence at the capsule $[6,7,9,11,13]$, and mini-open micro surgical resection is associated with less tissue destruction. If the tumor is a malignant schwannoma, the tumor may show invasion of neurovascular structures. In this situation, the surgery must be done with both the anterior and posterior microsurgical approaches.

To our knowledge, our case is the first one of an extremely large giant dumb-bell extradural lumbar schwannoma that was resected using mini-open microsurgical resection. This approach may be associated with minimal tissue destruction, translating into less blood loss and quicker functional recovery. However, further studies are needed to evaluate the relative efficacy and safety of minimally invasive resection of lumbar extremely large schwannomas. This approach may decrease the incidence of postoperative deformity, infection, neurovascular injury and eliminate the need for adjunctive fusion surgery. In addition, this study shows a need for modification of the classification (Kotil) system for global schwannomas.

\section{Conflict of Interest}

No potential conflict of interest relevant to this article was reported.

\section{References}

1. Sridhar K, Ramamurthi R, Vasudevan MC, Ramamurthi B. Limited unilateral approach for extramedullary spinal tumours. Br J Neurosurg 1998;12:430-3.

2. Celli P, Trillo G, Ferrante L. Spinal extradural schwannoma. J Neurosurg Spine 2005;2:447-56.

3. Jinnai T, Koyama T. Clinical characteristics of spinal nerve sheath tumors: analysis of 149 cases. Neurosurgery 2005;56:510-5.

4. Singh DK, Singh N, Rastogi M, Husain M. The transparaspinal approach: A novel technique for onestep removal of dumb-bell-shaped spinal tumors. J Craniovertebr Junction Spine 2011;2:96-8.

5. Love JG, Dodge HW Jr. Dumbbell (hourglass) neurofibromas affecting the spinal cord. Surg Gynecol Obstet 1952;94:161-72.

6. Satoh N, Ueda Y, Koizumi M, et al. Assessment of pure single nerve root resection in the treatment of spinal schwannoma: focus on solitary spinal schwannomas located below the thoracolumbar junction. J Orthop Sci 2011;16:148-55.

7. Lu DC, Dhall SS, Mummaneni PV. Mini-open removal of extradural foraminal tumors of the lumbar spine. J Neurosurg Spine 2009;10:46-50.

8. Park SC, Chung SK, Choe G, Kim HJ. Spinal intraosseous schwannoma: a case report and review. J Korean Neurosurg Soc 2009;46:403-8.

9. Ozawa H, Kokubun S, Aizawa T, Hoshikawa T, 
Kawahara C. Spinal dumbbell tumors: an analysis of a series of 118 cases. J Neurosurg Spine 2007;7:58793.

10. Sridhar K, Ramamurthi R, Vasudevan MC, Ramamurthi B. Giant invasive spinal schwannomas: definition and surgical management. J Neurosurg 2001;94:210-5.

11. Chiou SM, Eggert HR, Laborde G, Seeger W. Microsurgical unilateral approaches for spinal tumour surgery: eight years' experience in 256 primary operated patients. Acta Neurochir (Wien) 1989;100:127-33.

12. Seppala MT, Haltia MJ, Sankila RJ, Jaaskelainen JE, Heiskanen O. Long-term outcome after removal of spinal schwannoma: a clinicopathological study of 187 cases. J Neurosurg 1995;83:621-6.

13. Haji FA, Cenic A, Crevier L, Murty N, Reddy K. Minimally invasive approach for the resection of spinal neoplasm. Spine (Phila Pa 1976) 2011;36:E101826. 